\title{
Matter Creation via Vacuum Fluctuations in the Early Universe and Observed Ultra-High Energy Cosmic Ray Events
}

\author{
Vadim Kuzmin ${ }^{a, *}$ and Igor Tkachev ${ }^{a, b, \dagger}$ \\ ${ }^{a}$ Institute for Nuclear Research, Russian Academy of Sciences, \\ 60th October Anniversary Prosp. 7a, Moscow 117312, RUSSIA \\ ${ }^{b}$ Department of Physics, Purdue University, West Lafayette, IN 47907, USA
}

(September 28, 1998)

\begin{abstract}
Cosmic rays of the highest energy, above the Greisen-Zatsepin-Kuzmin cut-off of the spectrum, may originate in decays of superheavy long-living X-particles. These particles may be produced in the early Universe from vacuum fluctuations during or after inflation and may constitute a considerable fraction of Cold Dark Matter. We calculate numerically their abundance for a wide range of models. $\mathrm{X}$-particles are considered to be either bosons or fermions. Particles that are several times heavier than inflaton, $m_{\text {inflaton }} \approx 10^{13} \mathrm{GeV}$, and were produced by this mechanism, can account for the critical mass in the Universe naturally. In some cases induced isocurvature density fluctuations can leave an imprint in anisotropy of cosmic microwave background radiation.
\end{abstract}

PACS numbers: 98.70.Sa, 95.35.+d, 98.80.Cq

\section{INTRODUCTION}

According to the Greisen-Zatsepin-Kuzmin [1] (GZK) observation, the energy spectrum of Ultra High Energy (UHE) cosmic rays produced at extragalactic distances should exhibit an exponential cut-off at energy $E \sim 5 \times 10^{10} \mathrm{GeV}$. However, a number of cosmic ray events with energies well beyond the predicted GZK cutoff were recently observed by the various experimental groups [2]. This is an obvious contradiction with the standard cosmological and particle physics models and clearly requires some new physics beyond the Standard Model.

Conceptually, the simplest explanation [3,4] could be that the highest energy cosmic rays are produced in the decays of heavy long-living particles in cosmologically local part of the Universe. We will call these progenitors of the cosmic rays as X-particles. The mass of $X$-particles has to be very large, $m_{X} \gtrsim 10^{13} \mathrm{GeV}$, for them to be responsible for the cosmic rays events in the energy range $E \gtrsim 10^{11} \mathrm{GeV}$. It was noticed [5] [6] that such heavy particles are produced in the early Universe from the vacuum fluctuations and their abundance can be correct naturally, if the standard Friedmann epoch in the Universe evolution was preceded by the inflationary stage. Basically, it is the same process which during inflation had generated primordial large scale density perturbations and seeded formation of galaxies and galaxy clusters. However, there is a difference. Inflationary stage is not required to produce superheavy particles from the vacuum, unlike to the case of generation of the long wavelength perturbations. Rather, the inflation provides a cut off in excessive production of heavy particles which would happen in the Friedmann Universe if it would start from the initial singularity [5]. Note that we consider sterile or almost sterile $\mathrm{X}$-particles, so that their production in usual plasma interactions and decays can be neglected. Thermal production of heavy X-particles was discussed in Refs. [3, 1 , and production during reheating considered recently in [7].

Particle production from vacuum fluctuations in the Friedmann Universe during matter or radiation dominated stages had been considered long ago [8,9] and this gives the basis for simple estimates of X-particle abundance 15 which have the mass of oder or smaller than the Hubble constant at the end of inflation, $m_{X} \lesssim H_{i}$. However, the most interesting case of heavier X-particles, $m_{X}>H_{i}$, requires detailed calculations. Relevant calculations were done already by Chung, Kolb and Riotto [6]. However, to describe the end of inflation and the transition to the Friedmann universe, they match the fixed de Sitter background to the subsequent radiation or matter dominated expansion either as an instantaneous junction or with the help of a smoothing function. The result is especially sensitive to the details of the junction procedure in the case of large $m_{X}$ which is of interest for us here. Further, the range of models considered in Ref. [6] was restricted to the case of scalar particles with conformal coupling to gravity.

The purpose of the present paper is to calculate production of superheavy particles from the vacuum in the inflationary Universe for a wide range of models, and to give detailed and extended discussion of our other results already reported in letter [5]. Considering particle creation, we do not make any approximations. To this end we find numerically the exact evolution of the scale factor in the model of the "chaotic" inflation [11]. Our basic formalism relies on the method of Bogolyubov transformations, which for the case of particle creation by non-stationary gravitational field was developed in refs. [10,8]. We consider the possibility for X-particles to be either bosons or fermions.

In Section II we remind the reader about the explicit 
relations between mode functions of the field, coefficients of the Bogolyubov transformation and the particle number. In Section III we present results of our numerical calculations of particle production from the vacuum fluctuations both for Friedmann and inflationary stages in the universe evolution. In Section IV we relate results of previous section to the present-day density of X-particles. In Section $\mathrm{V}$ we discuss relevance of our results to the UHE cosmic rays events, and Section VI contains our conclusions.

\section{PARTICLE PRODUCTION BY A NON-STATIONARY GRAVITATIONAL FIELD}

Here we summarize the basic formalism of gravitational particle creation in an expanding universe which we employed in our analysis. For more details see Refs. 8,10. We choose the metric to be conformally flat at an early cosmological epoch, $d s^{2}=a(\eta)^{2}\left(d \eta^{2}-d \mathbf{x}^{2}\right)$. The number density of $X$-particles created from the vacuum in a time varying cosmological background can be written as

$$
n_{X}=\frac{1}{2 \pi^{2} a^{3}} \sum_{s} \int\left|\beta_{k}\right|^{2} k^{2} d k
$$

where $\beta_{k}$ are the Bogolyubov's coefficients which relate "in" and "out" mode functions, $k$ is the comoving momentum, and $\sum_{s}$ is the sum over spin states. The expression (11) gives the number density of particles only, with the equal amount of antiparticles being created in the case of charged fields. The creation of Bose and Fermi particles is to be considered separately.

i) Bosons. The mode functions, $\chi_{k} \equiv \chi_{k}(\eta)$ of a scalar Bose field are solutions of the oscillator equation

$$
\chi_{k}^{\prime \prime}+\omega_{k}^{2} \chi_{k}=0,
$$

with the time dependent frequency

$$
\omega_{k}^{2}=k^{2}-\frac{a^{\prime \prime}}{a}(1-6 \xi)+m_{X}^{2} a^{2} .
$$

Here $^{\prime} \equiv d / d \eta$. The constant $\xi$ describes direct coupling to the curvature, with $\xi=0$ corresponding to the minimal coupling and $\xi=1 / 6$ being the case of conformal coupling. Equations for massless conformally coupled quanta are reduced to the equations in flat space-time and such particles are not created. For massive particles conformal invariance is broken and particles are created regardless of value of $\xi$. Given the initial (vacuum) conditions

$$
\chi_{k}(0)=\omega^{-1 / 2}, \quad \chi_{k}^{\prime}(0)=-i \omega \chi_{k},
$$

the Bogolyubov's coefficients at any time moment $\eta$ are found to be

$$
\left|\beta_{k}\right|^{2}=\frac{\left|\chi_{k}^{\prime}\right|^{2}+\omega^{2}\left|\chi_{k}\right|^{2}-2 \omega}{4 \omega} .
$$

ii) Fermions. The relevant mode functions of the Fermi field satisfy the oscillator equation with the complex frequency

$$
\chi_{k}^{\prime \prime}+\left(\omega_{k}^{2}-i m_{X} a^{\prime}\right) \chi_{k}=0
$$

where the real part of the frequency is given by $\omega_{k}^{2}=$ $k^{2}+m_{X}^{2} a^{2}$. We choose

$$
\chi_{k}(0)=\sqrt{1-\frac{m_{X} a}{\omega}}, \quad \chi_{k}^{\prime}(0)=-i \omega \chi_{k},
$$

as the initial conditions. In this case we find per spin state

$$
\left|\beta_{k}\right|^{2}=\frac{\omega-m_{X} a-\operatorname{Im}\left(\chi_{k} \chi_{k}^{*^{\prime}}\right)}{2 \omega} .
$$

Summation over spins gives the factor of 2 in Eq. (1).

\section{NUMERICAL RESULTS}

\section{A. Power-Law Cosmology}

In this section we consider particle creation during the regular, i.e. non-inflationary, stage of the Universe expansion. We shall refer to this stage as the Friedmann stage of the Universe evolution. During the Friedmann stage the scale factor is given by the expression $a(t) \propto t^{\alpha}$ with $\alpha<1$. This case is of interest since $\alpha=1 / 2$ and $\alpha=2 / 3$ correspond to the radiation and matter dominated stages of the Universe expansion, which are inevitable. Moreover, in some cases (and we shall encounter such situations below) particle creation during this stage gives a dominant contribution, while creation during inflation (or at the transition between stages) is negligible.

Let us first consider massive particles conformally coupled to gravity. It is the particle mass which couples the system to the background expansion and serves as the source of particle creation. Therefore we expect $n_{X} \propto m_{X}^{3} a^{-3}$ at late times when particle creation diminishes and the number of particles conserves in a comoving volume. Because the scale factor has an arbitrary normalization, for definiteness it is more convenient to rewrite this expression using relations $a \propto(m t)^{\alpha}$, or $a \propto(m / H)^{\alpha}$. We prefer the latter choice since the exact value of time depends on the previous history in the inflationary cosmology and consequently the time coordinate, $t$, is ambiguously defined. Moreover, it is a non-zero value of the Hubble constant which is responsible for particle creation in an expanding universe. The particle production is expected to stop when $H \ll m_{X}$. Therefore, at the late epoch we can parameterize the anticipated formulae for $n_{X}$ as

$$
n_{X}=C_{\alpha} m_{X}^{3}\left(\frac{H}{m_{X}}\right)^{3 \alpha}
$$


where the constant $C_{\alpha}$ depends only upon the background cosmology, i.e. $\alpha$, and it can be found numerically. Note that for the radiation dominated case it was found in Ref. [8], $n_{X} \approx 5.3 \times 10^{-4} m_{X}^{3}\left(m_{X} t\right)^{-3 / 2}$. We confirm this result and extend it here to a broad range of cosmologically interesting values of $\alpha$, which includes the matter dominated case as well. Our results are summarized in Fig 1 where we plot $C_{\alpha}$ as a function of $\alpha$. The particle number reaches this asymptotic value, Eq. (9), if the initial vacuum state was defined at the sufficiently early epoch, $H(0) \gg m_{X}$. We found that $H(0) \approx 10^{4} m_{X}$ is sufficient for the results to become practically independent of the chose of the initial time if $\alpha=1 / 2$, with smaller (larger) ratio of $H(0) / m_{X}$ required at larger (smaller) $\alpha$.

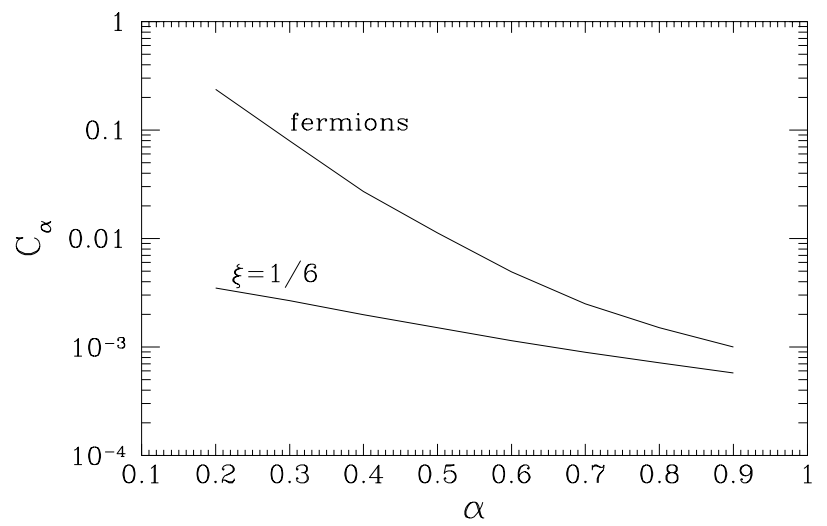

FIG. 1. Coefficient $C_{\alpha}$, defined in Eq. (9), is shown as a function of $\alpha$ for background cosmology with a power law scale factor $a \propto t^{\alpha}$.

For the radiation dominated Universe one finds $\Omega_{X} \equiv$ $\rho_{X} / \rho_{c}=m_{X} n_{X} 32 \pi G t^{2} / 3$ with the present value of $\Omega_{X}$ being equal to $\Omega_{X} \sim 2 \times 10^{-2}\left(m_{X}^{2} / M_{\mathrm{Pl}}^{2}\right) \sqrt{m_{X} t_{e}}$, where $t_{e}$ is the time of equal densities of radiation and matter in the $\Omega=1$ Universe. This gives $\Omega_{X} \sim\left(m_{X} / 10^{9} \mathrm{GeV}\right)^{5 / 2}$. We see that stable particles with $m_{X} \gtrsim 10^{9} \mathrm{GeV}$ will overclose the Universe even if initially they were in a vacuum state and were created from the vacuum during the regular Friedmann radiation dominated stage of the evolution. (It is possible to separate the vacuum creation from the creation in collisions in plasma since X-particles may be effectively sterile.)

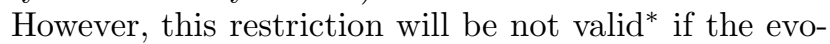
lution of the Universe, as it is believed, was more complicated than the simple radiation dominated expansion from a singularity. The Hubble constant may have never exceeded $m_{X}$, which is the case of inflation, $H(0) \approx$ $m_{\phi} \approx 10^{13} \mathrm{GeV}$. Moreover, compared to the case considered above, the density of X-particles created during inflation is additionally diluted by the entropy release in

${ }^{*}$ Another possibility is the late time entropy release. reheating after inflation.

\section{B. Inflationary Cosmology}

Any viable modern cosmological model invokes the hypothesis of inflation (for a review and list of references, see refs. 12, 13). During inflation the Universe expands exponentially which solves the horizon and flatness problems of standard Big-Bang cosmology. Inflation is generally assumed to be driven by the special scalar field $\phi$ known as the inflaton. Fluctuations generated at inflationary stage may have the strength and the power spectrum suitable for generation of the large-cale structure. This fixes the range of parameters of the inflaton potential. For example, the mass of the inflaton field has to be $m_{\phi} \sim 10^{13} \mathrm{GeV}$. During inflation, the inflaton field slowly rolls down towards the minimum of its potential. Inflation ends when the potential energy associated with the inflaton field becomes smaller than the kinetic energy. Coherent oscillations of the inflaton field contained all the energy of the Universe at that time. It is possible [14 that a significant fraction of this energy was released to other boson species after only a dozen oscillations of the inflaton field, in the regime of a broad parametric resonance. This process was studied in detail [15]16]. It was shown that even rather heavy particles with masses by an order of magnitude larger than the inflaton mass can be produced quite copiously (see also [17]). Applying these results to the case of our interest, we find that the stable very heavy particles, $m_{\phi} \lesssim m_{X} \lesssim 10 m_{\phi}$, will be generally produced in excess in this process.

However, if the parametric resonance is ineffective for some reason, and one estimates the particle number density after inflation at the level of initial conditions used in Refs. [15], one finds that $\Omega_{X}$ might prove to be of the acceptable magnitude. This level is saturated by the fundamental process of particle creation during inflation from vacuum fluctuations and it is the same process which generated primordial large scale density perturbations. Parametric resonance for $X$ particles is turned off if $X$ field is either a fermion field or its coupling to inflaton is small, $g^{2} \ll 10^{4}\left(m_{X} / m_{\phi}\right)^{4}\left(m_{\phi} / M_{\mathrm{Pl}}\right)^{2}$ [15]. (Parametric creation of fermions was considered in Refs. [18]. While it is not a "resonance" process, it might be useful for creation of very heavy fermions.)

Particle creation from vacuum fluctuations during inflation (or in the de Sitter space) was extensively studied [19,20], usually in the case of small $m_{X}$ and in application to generation of density fluctuations necessary for the large scale structure formation. The characteristic quantity which is usually cited, the variance of the field, $\left\langle X^{2}\right\rangle$, is defined by an expression similar to Eq. (1). In the typical case the difference is given by the factor $2 \sin ^{2}\left(\omega_{k} \eta\right) / \omega_{k}$ in the integrand. For example, for the scalar Bose field with the minimal coupling to the curvature, $\left\langle X^{2}\right\rangle=3 H_{i}^{4} / 8 \pi^{2} m_{X}^{2}$ if $m_{X} \ll H_{i}$ [19,20]. For 
massless self-interacting field $\left\langle X^{2}\right\rangle \approx 0.132 H_{i}^{2} / \sqrt{\lambda}$ [21. Particle creation in the settings close to our problem, but for the specific case of the Hubble dependent effective mass, $m_{X}(t) \propto H(t)$, was considered in Ref. [22].

In general, if $m_{X} \sim H_{i} \approx m_{\phi}$, we can again expect [5] that the particle density will be given by Eq. (9). In general, the coefficient $C$ in this equation will be a function of the ratio $H_{i} / m_{X}$, of the coupling constant $\xi$, and will depend on details of the transition between inflationary and matter (or radiation) dominated phases, etc. For $m_{X}>H_{i}$ an exponential suppression is expected. Detailed numerical study of X-particle abundance created from the vacuum in inflationary cosmology is the subject of this Section of the paper.

The case of large $m_{X}$ with application to the dark matter problem was already considered in Ref. [6]. However, the analysis was restricted to the case of conformally coupled scalar particles only, and approximations were made to the extent that the fixed de Sitter background was matched to the subsequent radiation or matter dominated expansion either as an instantaneous transition or with the help of some smoothing function. Particle creation in the case of large $m_{X}$ can be especially sensitive to (some extent arbitrary) details of such junction procedure.

In the present paper, considering particle creation as described by Eqs. (11)-(8), we do not make any approximations. We assume a specific model of inflation, namely "chaotic" inflation [11], and find numerically the exact evolution of the scale factor in this model. We consider both the "massive" inflaton with the scalar potential $V(\phi)=m^{2} \phi^{2} / 2$, and the "massless" inflaton with the potential $V(\phi)=\lambda \phi^{4} / 4$. The normalization to the observable large scale structure requires $m^{2} / M_{\mathrm{Pl}}^{2} \approx 10^{-12}$ in the former model and $\lambda \approx 10^{-13}$ in the latter model. We consider creation of conformally and minimally coupled scalar X-particles, as well as creation of X-fermions.

Our results are summarized in Fig. 2 (massive inflaton) and Fig. 3 (massless inflaton). We define the energy density in X-particles at late times as $\rho_{X}=m_{X} n_{X}$. In the case of the massive inflaton we normalized $\rho_{X}$ by the total energy density. The ratio of those two quantities reaches some asymptotic value and then remains constant in a matter dominated universe, and can be measured at any sufficiently late time in the numerical simulation which does not include the inflaton decay. For each value of $m_{X}$ we integrated equations (11)-(8) until the asymptotic constant value of this ratio was reached. Similarly, the convenient quantity to be measured in the massless inflaton model is proportional to $\rho_{X} / \rho^{3 / 4}$, which also becomes constant at late times. The curves labeled by $\xi=0$ and $\xi=1 / 6$ correspond to the minimal and the conformal coupling to gravity, respectively. The dashed line describes creation of fermions.

We see that particle production is most effective in the case of scalar particles with minimal coupling, exceeding $\rho_{X}$ generated in other models by many orders of magnitude if $m_{X} \lesssim m$. Note also that the ratio of the energy density in $X$-particles to the total energy density is independent upon $m_{X}$ at $m_{X}<m$ in this model. This might seem as easily understandable. Indeed, magnitude of fluctuations generated during inflation in de Sitter space is $\left\langle X^{2}\right\rangle \propto m_{X}^{-2}$ if $m_{X} \ll H_{i}$. Multiplying this by $m_{X}^{2}$ one would find $\rho_{X} \approx m_{X}^{2}\left\langle X^{2}\right\rangle=$ const, seemingly in agreement with observed behavior, Fig. 2 However,this agreement is rather coincidental since it is possible to relate $\rho$ and $\left\langle X^{2}\right\rangle$ in this simple manner at late times only.

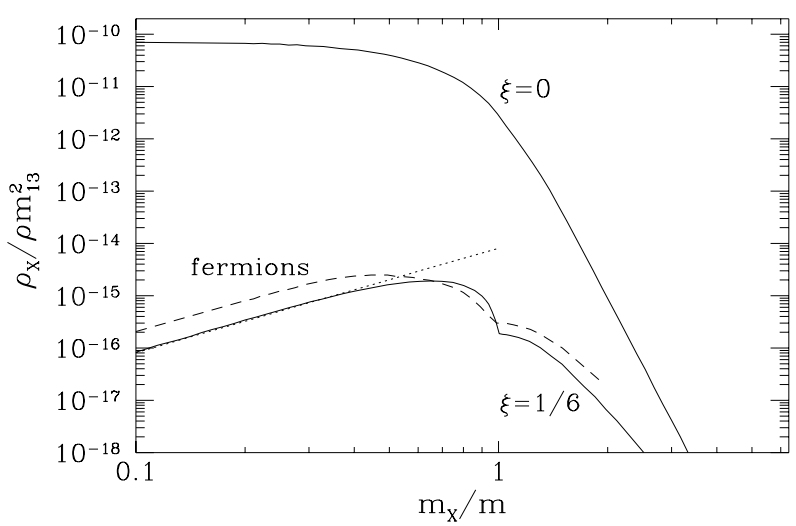

FIG. 2. Ratio of the energy density in $X$-particles to the total energy density at late times in a model with the massive inflaton, $V(\phi)=m^{2} \phi^{2} / 2$, as a function of $\mathrm{X}$ particle mass, $m_{X}$. For the inflaton mass we defined $m_{13} \equiv m / 10^{13} \mathrm{GeV}$. The dotted line is the low mass asymptotic, Eq. (9).

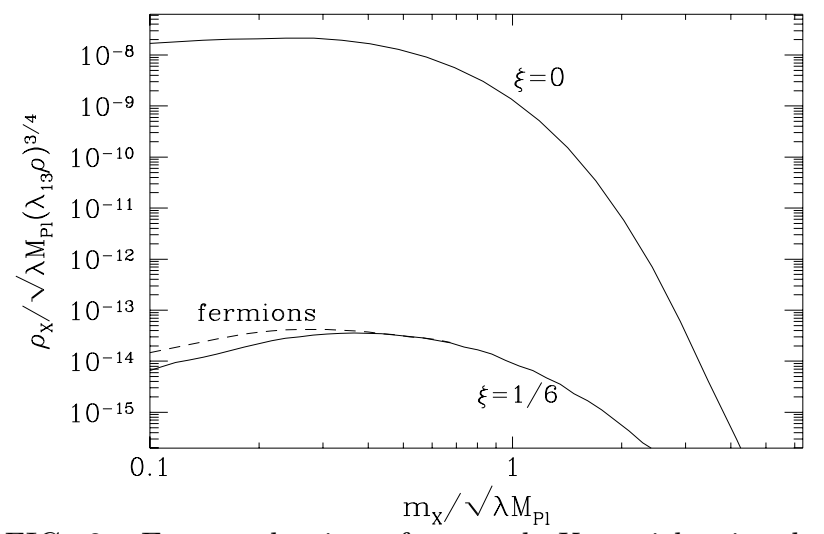

FIG. 3. Energy density of created $X$-particles in the massless inflaton model, $V(\phi)=\lambda \phi^{4} / 4$. We defined $\lambda_{13} \equiv \lambda / 10^{-13}$.

In our numerical calculations we had found that the variance $\left\langle X^{2}\right\rangle$ of the field $X$ measured at the end of inflation is independent upon $m_{X}$ if the mass of $X$ is small. At some later epoch when $H \approx m_{X}$, which will be long after the end of inflation if $\mathrm{X}$ is a light field, the field $X$ starts to oscillate on all scales, including $k=0$. Only at this time, which we denote by $t_{X}$, all field fluctuations are transformed into non-zero particle density and we can use $\rho_{X}=m_{X} n_{X} \approx m_{X}^{2}\left\langle X^{2}\right\rangle$. The variance of $X$ fluctuations 
was unchanged on large scales, starting from the end of inflation down to the time $t_{X}$. So, when the field starts to oscillate, $\rho_{X} \propto m_{X}^{2}$. However, the energy density of the inflaton field, $\rho=3 H^{2} / 8 \pi G$ decreased during this time interval in proportion to $H^{2}\left(t_{X}\right) / H^{2}(0) \approx m_{X}^{2} / H^{2}(0)$. That is why the ratio of the energy density in $X$-particles to the total energy density does not depends on $m_{X}$ when measured at $t>t_{X}$.

Variance of the field $\mathrm{X}$ is different from the usually calculated for the fixed de Sitter inflationary background because we consider the actual evolution of the scale factor and the value of the Hubble parameter is not constant during inflation, being larger at earlier times. Correspondingly, the number of created particles per decade of $k$ grows logarithmically towards small $\mathrm{k}$ if $m_{X}$ is small. (Power spectrum behaves similarly.) For definiteness, we restricted our calculations to $\sim 10^{15}$ for the expansion factor during inflation. The examples of the particle number, $k^{3} n_{X}(k)$ for several values of $m_{X}$ are shown in Fig. 4 at the moment corresponding to 10 completed inflaton oscillations. The particle momentum is measured in units of the inflaton mass. In contrast to this, in fixed de Sitter background $4 \pi^{2}\left\langle X^{2}\right\rangle \approx H^{2} \int d \ln k(k / H)^{3-2 \nu}$ with $3-2 \nu \approx 2 m_{X}^{2} / 3 H^{2}$ at small $m_{X}$ and consequently $\left\langle X^{2}\right\rangle \propto 1 / m_{X}^{2}$. When our code was tested on fixed de Sitter background it reproduced the proper power spectrum exactly. Note that the power spectrum in fixed de Sitter background grows towards large values of $k$, which is opposite to the behavior of Fig. 4 .

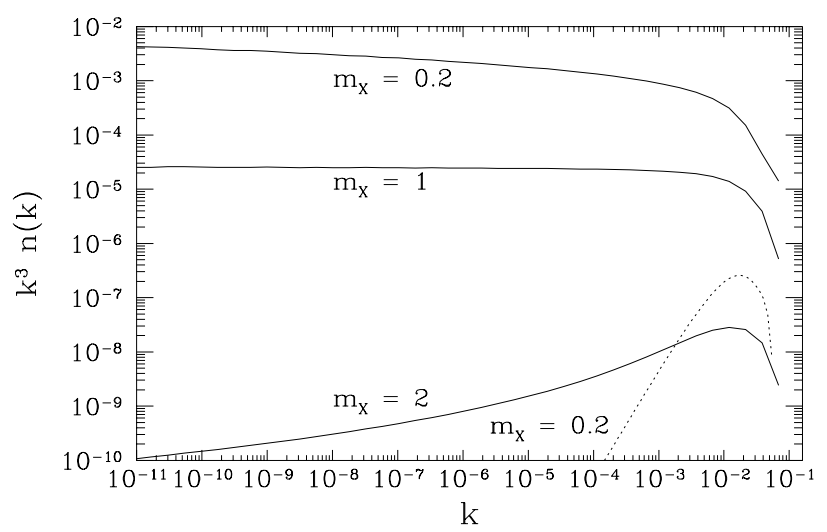

FIG. 4. Spectrum of created particles, $k^{3} n(k)$, in a model with massive inflaton is shown for several choices of the mass of scalar X-particle with the minimal coupling (solid lines) and the conformal coupling (dotted line). Masses and momenta, $k$, are given in units of the inflaton mass.

Therefore, calculations which would be based on customary procedure of matching a fixed de Sitter background to a subsequent Friedmann stage would give wrong results in this case. Note, however, that the result depends upon duration of inflationary stage, so our results in the case of scalars with minimal coupling and $m_{X}<m$ should be considered only as a lower bound on $\mathrm{X}$-particle abundance.
Matching is also dangerous in the case of large $m_{X}$, and its influence was studied in detail in Ref. [6]. When the change is too abrupt, it generates artificial particles. This can easily happen for $m_{X}>m$, see e.g. 23] where excessive production was found. At $m_{X} \gtrsim m$ the number of created particles decreases exponentially with $m_{X}$. For the case of massive inflaton our result shown in Fig. 2 can be fitted as

$$
\frac{\rho_{X}}{\rho} \approx 10^{-10} \frac{m_{X}}{m} m_{13}^{2} \exp \left(-2 \pi m_{X} / m\right),
$$

where $m_{13} \equiv m / 10^{13} \mathrm{GeV}$. (Note that the fitting formulae is approximate and arbitrary to some extent).

As Fig. 1 shows, the power spectrum of fluctuations in $\mathrm{X}$-particles is almost scale independent at small $\mathrm{k}$ if $m_{X} / m \approx 1$. Therefore, if such particles constitute a considerable fraction of dark matter, this fluctuations will be transformed into isocurvature density perturbations at late times and can affect large scale structure formation. Isocurvature fluctuations produce 6 times larger angular temperature fluctuations in cosmic microwave background radiation (CMBR) for the same amplitude of long-wavelength density perturbations compared to the adiabatic case [24]. Therefore, to fit observations by a single spectrum, the mass fluctuation spectrum in isocurvature cold dark matter cosmology must be tilted (with respect to scale invariant spectrum) to favor smaller scales.

Let the power spectrum of the field fluctuations be $k^{3} P_{X}(k) \propto k^{\beta}$. To fit to the second moments of the largescale mass and (not contradict to) cosmic microwave background distributions requires $\beta \gtrsim 0.25$. Models with $\beta$ ranging from 0.3 to 0.6 were constructed and considered in Refs. 25,26. It is interesting that in our case the spectrum is indeed correctly tilted, see Fig. A, for $m_{X}$ which is somewhat larger than the inflaton mass, On the smallest scale of our integration, $k \approx 10^{-15}$ we find $\beta \approx 0.1$ for $m_{X} / m=2$ and $\beta \approx 0.3$ for $m_{X} / m=3$. Since $\beta$ decreases with the length scale increasing, on scales corresponding to the current horizon $\beta$ will be smaller. Because of that the amplitude of perturbations will be also too small on cosmological scales when $\beta$ will be reaching desired magnitude, $\beta \gtrsim 0.25$. Indeed, density fluctuations, $\delta \rho_{X} / \rho_{X}$, which are roughly proportional to spectra shown in Fig. 4, are reaching unity near the break in the power spectrum ( $k \sim 1$ at the end of inflation) and on the scales of interest amplitude of fluctuations became too small already at $m_{X} / m=3$. However, their magnitude may be just right, $\delta \rho_{X} / \rho_{X} \approx 10^{-5}$, on scales $k \gtrsim 10^{-25}$ for the case $m_{X} / m \approx 2$ (or slightly larger) if $\Omega_{X} \approx 1$, see Fig. \#. Magnitude can not correspond to the observable on the galaxy scales since then there will be too much fluctuations in CMBR. This gives a constraint on the models. X-particles with minimal coupling to gravity, $m_{X}>2 m$ and $\Omega_{X} \approx 1$ are excluded. (Such particles with small contribution to $\Omega$ may exist though.) However, the magnitude of the density fluctuations induced in the process of $\mathrm{X}$ particle creation can 
correspond to the observable on the horizon scale, and be responsible for fluctuations in CMBR. X-particles with $m_{X} / m \approx 2$ and $\Omega_{X} \approx 1$ can give such contribution naturally. (Source for the galaxies seeds has to be different in this case.) Note that the density autocorrelation function is proportional to the square of the field autocorrelation function in the present case, and the density fluctuations are non-Gaussian.

In the case of fermions or scalar particles with conformal coupling, the number density of created particles has a kink at $m_{X}=m$ in the model with massive inflaton, see Fig. 2. This signifies that different mechanisms are responsible for particle creation at $m_{X}>m$ and $m_{X}<m$. Particles heavier than the inflaton are created during inflation, while particles lighter than the inflaton are created after inflation during the regular Friedmann stage of expansion. We see that particle creation during this latter stage is actually more effective $\left(n_{X}\right.$ rapidly increases when $m_{X}$ drops slightly below $m$ ). Hence, we can assume that the number density of created particles at $m_{X} \ll m$ is given by Eq. (9) with $C_{\alpha}=C_{2 / 3} \cdot\left(C_{2 / 3} \approx 9.7 \times 10^{-4}\right.$ for the conformal scalars, see Fig. 1.) Since the total energy density is given by $\rho=3 H^{2} / 8 \pi G$, we find for $\alpha=2 / 3$

$$
\frac{\rho_{X}}{\rho}=\frac{8 \pi}{3} \frac{m_{X}^{2}}{M_{\mathrm{Pl}}^{2}} C_{3 / 2} .
$$

This function is plotted in Fig. 2 by the dotted line which shows that already at $m_{X}<0.5 \mathrm{~m}$ the number density of created particles is given by Eq. (9), indeed. Note that in Ref. [6] all particle creation was attributed to the transition from the inflationary to matter dominated phase, while we find that for $m_{X}<m$ the dominant particle creation occurs at a later stage, when $H \approx k \approx$ $m_{X}$ (for $m_{X}<m$ ). The correct interpretation allows us to identify the low mass asymptotic, Eq. (11), see also 5.

Scalar particles with minimal coupling to gravity are produced predominantly during the inflationary stage for all $m_{X}$, and the resulting number density is much higher, see Fig. 2.

Conformal coupling, $\xi=1 / 6$, is a stable point of renormalization group equations. Otherwise $\xi$ is a running coupling constant and there is no reason to expect the coupling to be, say, minimal at mass scales of inflation. The dependence of X-particle abundance on the coupling to the curvature scalar is shown in Fig. 5 .

The situation in the case of the massless inflaton, $V(\phi)=\lambda \phi^{4} / 4$, is quite similar. However, now there is no distinctive mass scale in the equations of motion for the scale factor, and the kink in the function $n_{X}\left(m_{X}\right)$ does not appear. The quantity $\sqrt{\lambda} \phi$ might be considered as an effective inflaton mass, but it changes during the evolution. For definiteness we plot $n_{X}$ as a function of $m_{X} / \sqrt{\lambda} M_{\mathrm{Pl}}$. Note that the transition from the inflationary to the radiation dominated stage occurs at $\phi \approx 0.35 M_{\mathrm{Pl}}$ [15] and this may define the more natural mass scale for the ratio $m_{X} / m_{\mathrm{eff}}(\phi)$. Indeed, we observe that $n_{X}$ reaches its maximum when $m_{X} \approx 0.35 \sqrt{\lambda} M_{\mathrm{Pl}}$, see Fig. 3 .

We find that the number density of produced fermions in both cases is approximately equal to the number density of scalar particles with conformal coupling. The main difference amounts to a spin factor of two.

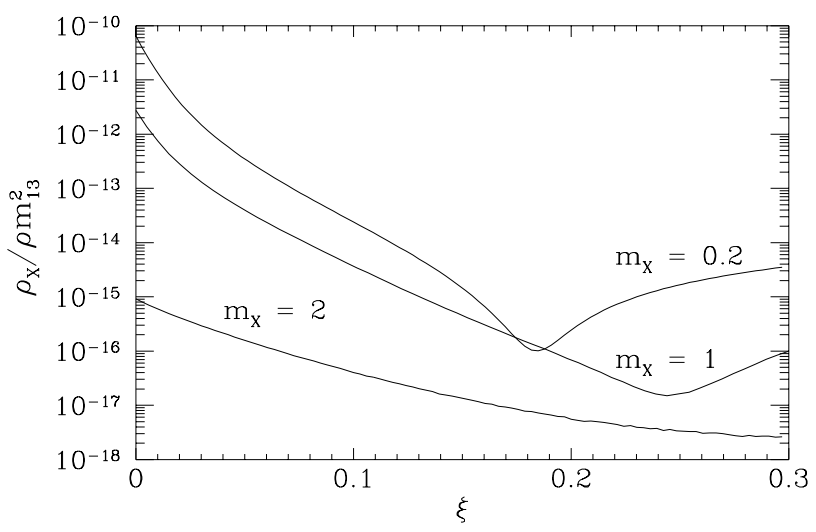

FIG. 5. Ratio of the energy density in $X$-particles to the total energy density at late times in a model with the massive inflaton, $V(\phi)=m^{2} \phi^{2} / 2$, as a function of $\xi$.

\section{PRESENT DENSITY OF X-PARTICLES}

Let us estimate the present day number density of Xparticles. Consideration is somewhat different for massive and massless inflaton models.

1. Massive inflaton, $V(\phi)=m_{\phi}^{2} \phi^{2} / 2$.

In this case the inflation is followed by the stage of matter domination which lasts until inflaton decays and thermal equilibrium is established. Let us denote the temperature at the beginning of radiation dominated stage as $T_{*}$. Actually, a transition to the radiation dominated universe does not necessarily occur with the final state being in thermal equilibrium. Complications arise if there are light bosons in a theory, $m_{B} \ll m_{\phi}$, even relatively weakly coupled to the inflaton, $g^{2} \gtrsim 10^{4} m_{\phi}^{2} / M_{\mathrm{Pl}}^{2} \sim 10^{-8}$. In this case the inflaton will decay via parametric resonance after just a several oscillations and relativistic degrees of freedom start to dominate the equation of state. This happens typically when the energy density in the inflaton oscillations is red-shifted by a factor $r \approx 10^{-6}$ compared to the value $m_{\phi}^{2} M_{\mathrm{Pl}}^{2}$ 11, 15 . Matter is still far from being in thermal equilibrium, but since Universe expansion already at this point changes to radiation dominated it is convenient to characterize this radiation dominated stage by an equivalent "temperature", $T_{*} \sim r^{1 / 4} \sqrt{m_{\phi} M_{\mathrm{Pl}}}$. In any case, $T_{*}$ is the model dependent parameter and we leave it as such.

Until the moment when the equation of state changes from matter dominated to radiation dominated, the ratio of the energy density in X-particles to the total energy density retains its value reached at the end of inflation, 
$\rho_{X} / \rho=$ const. This ratio is plotted in Fig. 2 for different models as a function of model parameters and it defines the present day ratio of $\rho_{X}$ to the critical density in the Universe, $\rho_{c}$, as follows:

$$
\Omega_{X} h^{2}=\frac{\rho_{X}}{\rho} \frac{T_{*}}{\gamma T_{0}} \Omega_{R} h^{2},
$$

where $T_{0}$ is present temperature of cosmic microwave background, and $\Omega_{R} h^{2}=\rho_{\text {rad }} h^{2} / \rho_{c} \approx 4.31 \times 10^{-5}$ is the fraction of critical density that is in radiation today [13]. The following definitions are used here $\rho_{c}=3 H_{0}^{2} M_{\mathrm{Pl}}^{2} / 8 \pi$ and $H_{0}=100 h \mathrm{~km} \mathrm{sec}^{-1} \mathrm{Mpc}^{-1}$. Parameter $\gamma$ describes the change in entropy per comoving volume for the whole cosmological history starting from $T=T_{*}$ down to the present day, i.e. $s_{0} \equiv \gamma s_{*}$. In simple cosmological models, $\gamma \sim 1$. However, in models with subsequent latent heat release (due to phase transitions or due to decay of long living particles, e.g. moduli fields) $\gamma$ can be very large.

Let us assume for illustration purposes that $T_{*}=10^{9}$ $\mathrm{GeV}$, i.e. $\left(T_{*} / T_{0}\right)\left(\Omega_{R} h^{2}\right) \approx 1.8 \times 10^{17}$. (This is the highest allowed reheating temperature when gravitinos in supergravity theories are not overproduced.) With $\gamma \sim 1$ we find that $\Omega_{X} h^{2} \sim 1$ if $m_{X} / m \approx 2$ in models with scalar X-particles with conformal coupling, or if Xparticles are fermions, see Fig. 2. For minimally coupled scalars this value of $\Omega_{X}$ is reached for $m_{X} / m \approx 3$.

To summarize this subsection: $\Omega_{X} h^{2}$ is found multiplying the data of Fig. 2 by a factor $1.8 \times$ $10^{17}\left(T_{*} / \gamma 10^{9} \mathrm{GeV}\right) m_{13}^{2}$.

1. Massless inflaton, $V(\phi)=\lambda \phi^{4} / 4$.

Before and after the decay of the inflaton the Universe expands as radiation dominated. In Section III for the state which precedes the decay of inflaton oscillations we had calculated the quantity $\rho_{X} / \sqrt{\lambda} M_{\mathrm{Pl}} \rho^{3 / 4}$, where $\rho$ is the inflaton energy density. After the decay of the inflaton, in a state of thermal equilibrium we can use

$$
s_{*}=\frac{4}{3}\left(\frac{\pi^{2} g_{*}}{30}\right)^{1 / 4} \rho_{*}^{3 / 4},
$$

where $g_{*}$ is the number of relativistic degrees of freedom at temperature $T_{*}$. Assuming instantaneous transition between those states, $\rho_{*}=\rho$, and again using $\Omega_{X}=$ $\left(\rho_{X} / T_{0} s_{0}\right)\left(T_{0} s_{0} / \rho_{c}\right)$ together with $s_{0} \equiv \gamma s_{*}$, we find

$$
\Omega_{X} h^{2}=\frac{\sqrt{\lambda} M_{\mathrm{Pl}}}{\gamma T_{0}}\left(\frac{\pi^{2} g_{*}}{30}\right)^{-1 / 4} \frac{\rho_{X}}{\sqrt{\lambda} M_{\mathrm{Pl}} \rho^{3 / 4}} \Omega_{R} h^{2} .
$$

We see that $\Omega_{X} h^{2}$ can be found by multiplying the data plotted in Fig. 3 by a factor $2.5 \times 10^{20} \lambda_{13}^{5 / 4} / \gamma g_{200}^{1 / 4}$, where we defined $g_{200} \equiv g_{*} / 200$, and used $\sqrt{\lambda} M_{\mathrm{Pl}} / T_{0} \approx 1.6 \times$ $10^{25} \sqrt{\lambda_{13}}$. Note that $\sqrt{\lambda} M_{\mathrm{Pl}} \approx 3.9 \times 10^{12} \sqrt{\lambda}_{13} \mathrm{GeV}$, so that already with $\gamma=1$ we shall have $\Omega_{X}<1$ if $m_{X} \gtrsim 4 \times 10^{13} \mathrm{GeV}$.

\section{X-PARTICLES AND UHE COSMIC RAYS.}

Why were the Ultra High Energy cosmic rays observed above the Greisen-Zatsepin-Kuzmin energy cut-off? One solution to the problem might be provided, for example, by the existence of some exotic particles which are able to propagate (evading the GZK bound) from cosmological distances and yet interact in the Earth's atmosphere like a hadron. A particle with such conflicting properties was found in a class of supersymmetric theories [27]. Alternatively, high energy cosmic rays may have been produced locally within the GZK distance. One possibility is connected with the destruction of topological defects 28], while another one is connected with decays of primordial heavy long-living particles [3, 4]. The candidate $X$-particle must obviously obey constraints on mass, density and lifetime.

In order to produce cosmic rays in the energy range $E \gtrsim 10^{11} \mathrm{GeV}$, the mass of $X$-particles has to be very large, $m_{X} \gtrsim 10^{13} \mathrm{GeV}$ [3]:4]. The lifetime, $\tau_{X}$, cannot be much smaller than the age of the Universe, $\tau \approx 10^{10} \mathrm{yr}$. With such a short lifetime, the observed flux of UHE cosmic rays will be generated with the rather low density of $X$-particles, $\Omega_{X} \sim 10^{-12}$, where $\Omega_{X} \equiv m_{X} n_{X} / \rho_{\text {crit }}$, $n_{X}$ is the number density of $\mathrm{X}$-particles and $\rho_{\text {crit }}$ is the critical density. On the other hand, X-particles must not overclose the Universe, $\Omega_{X} \lesssim 1$. With $\Omega_{X} \sim 1$, the $\mathrm{X}$-particles may play the role of cold dark matter and the observed flux of UHE cosmic rays can be matched if $\tau_{X} \sim 10^{22} \mathrm{yr}$. The allowed windows are quite wide [3], but on the exotic side, which may give rise to some problems.

The possibility that X-particles were generated from the vacuum by gravitational interactions [5.6] may answer to the question why their abundance should lie in the interesting range $\Omega_{X} \lesssim 1$ and was considered in detail in the present paper.

The problem of the particle physics mechanism responsible for a long but finite lifetime of very heavy particles can be solved in several ways. For example, otherwise conserved quantum number carried by X-particles may be broken very weakly due to instanton transitions [3], or quantum gravity (wormhole) effects [4]. If instantons are responsible for $X$-particle decays, the lifetime is estimated as $\tau_{X} \sim m_{X}^{-1} \cdot \exp \left(4 \pi / \alpha_{X}\right)$, where $\alpha_{X}$ is the coupling constant of the relevant gauge interaction. The lifetime will fit the allowed window if the coupling constant (at the scale $m_{X}$ ) is $\alpha_{X} \approx 0.1$ [3].

A class of natural candidates for superheavy long-living particles which arise in string and $M$ theory was reevaluated recently in Ref. [30] and particles with desired mass and long life-time were identified. Another interesting candidates were found among adjoint messengers in gauge mediated supergravity models [6, 31. 


\section{CONCLUSIONS}

We have shown that the very weakly interacting superheavy X-particles with $m_{X}=($ a few $) \cdot 10^{13} \mathrm{GeV}$ may naturally constitute a considerable fraction of Cold Dark Matter. These particles are produced in the early Universe from vacuum fluctuations during or after inflation. Related density fluctuations may have left an imprint in fluctuations of cosmic microwave background radiation if scalar X-particles with minimal coupling to gravity are approximately twice heavier than the inflaton and $\Omega_{X} \sim 1$. Decays of X-particles may explain UHE cosmic rays phenomenon.

Our hypothesis has unique observational consequences. If UHE cosmic rays are indeed due to the decay of these superheavy particles, there has to be a new sharp cut-off in the cosmic ray spectrum at energies somewhat smaller than $m_{X}$. Since the number density $n_{X}$ depends exponentially upon $m_{X} / m_{\phi}$, the position of this cut-off is fixed and can be predicted to be near $m_{\phi} \approx 10^{13} \mathrm{GeV}$, the very shape of the cosmic ray spectrum beyond the GZK cut-off being of quite generic form following from the QCD quark/gluon fragmentation. Next generation experiments, like the Pierre Auger Project [32], High Resolution Fly's Eye [33], the Japanese Telescope Array Project [34], may prove to be able to discover this fundamental phenomenon.

We conclude that the observations of Ultra High Energy cosmic rays can probe the spectrum of elementary particles in the superheavy range and can give an unique opportunity for investigation of the earliest epoch of evolution of the Universe, starting with the amplification of vacuum fluctuations during inflation through fine details of gravitational interaction and down to the physics of reheating.

We are grateful to S. Khlebnikov, A. Linde and A. Riotto for useful discussions. V. A. Kuzmin and I. I. Tkachev thank Theory Division at CERN for hospitality where this work was initiated. The work of V. K. was supported in part by Russian Foundation for Basic Research under Grant 95-02-04911a. I. I. T. was supported in part by the U.S. Department of Energy under Grant DE-FG02-91ER40681 (Task B) and by the National Science Foundation under Grant PHY-9501458.

[1] K. Greisen, Phys. Rev. Lett. 16, 748 (1966); G. T. Zatsepin and V. A. Kuzmin, Pisma Zh. Eksp. Teor. Fiz. 4, 114 (1966).

[2] N. Hayashida et. al., Phys. Rev. Lett. 73, 3491 (1994); D. J. Bird et. al., Astroph. J. 424, 491 (1994); 441, 144 (1995); T. A. Egorov et. al., in: Proc. Tokyo Workshop on Techniques for the Study of Extremely High Energy Cosmic Rays, ed. M.Nagano (ICRR, U. of Tokyo, 1993).
[3] V. A. Kuzmin and V. A. Rubakov, astro-ph/9709187.

[4] V. Berezinsky, M. Kachelriess and A. Vilenkin, Phys. Rev. Lett. 79, 4302 (1997).

[5] V. A. Kuzmin and I. I. Tkachev, hep-ph/9802304.

[6] D. J. H. Chung, E. W. Kolb and A. Riotto, hepph/9802238; hep-ph/9805473.

[7] D. J. H. Chung, E. W. Kolb and A. Riotto, hep$\mathrm{ph} / 9809453$.

[8] S. G. Mamaev, V. M. Mostepanenko, and A. A. Starobinskii, ZhETF 70, 1577 (1976) [ Sov. Phys. JETP 43, 823 (1976)].

[9] S. G. Mamaev, V. M. Mostepanenko, and V. M. Frolov, Yad. Fiz. 23, 1118 (1996), ibid 26, 215 (1997).

[10] L. Parker, Phys. Rev. 183, 1057 (1969); A. A. Grib and S. G. Mamaev, Yad. Fiz. 10, 1276 (1969) [Sov. J. Nucl. Phys. 10, 722 (1970)]; Ya. B. Zel'dovich and A. A. Starobinslii, Zh. Exp. Theor. Fiz. 61, 2161 (1971) [Sov. Phys. JETP 34, 1159 (1972)].

[11] A. D. Linde, Phys. Lett. 108B (1982) 389; A. Albrecht and P. J. Steinhardt, Phys. Rev. Lett. 48 (1982) 1220.

[12] A. D. Linde, Particle Physics and Inflationary Cosmology, (Harwood Academic, New York, 1990).

[13] E. W. Kolb and M. S. Turner, The Early Universe, (Addison-Wesley, Reading, Ma., 1990).

[14] L. A. Kofman, A. D. Linde and A. A. Starobinsky, Phys. Rev. Lett. 73, 3195 (1994); Y. Shtanov, J. Traschen, and R. Brandenberger, Phys. Rev. D 51, 5438 (1995).

[15] S. Yu. Khlebnikov and I. I. Tkachev, Phys. Rev. Lett. 77, 219 (1996); Phys. Lett. B390, 80 (1997); Phys. Rev. Lett. 79, 1607 (1997); Phys. Rev. D56, 653 (1997).

[16] L. A. Kofman, A. D. Linde and A. A. Starobinsky, Phys. Rev. D56, 3258 (1997).

[17] E. W. Kolb, A. Riotto, and I. I. Tkachev, Phys. Lett. B423, 348 (1998).

[18] J. Baacke, K. Heitmann, and C. Patzold, hepph/9806205; P. B. Greene and L. Kofman, hep$\mathrm{ph} / 9807339$.

[19] N. A. Chernikov and E. A. Tagirov, Ann. Inst. Henri Poinare 9A, 109 (1968); E. A. Tagirov, Ann. Phys. 76, 561 (1973); T. S. Bunch and P. C. W. Davies, Proc. R. Soc. A360, 117 (1978).

[20] A. D. Linde, Phys. Lett. 116B, 335 (1982); A. A. Starobinsky, Phys. Lett. 117B, 175 (1982); A. Vilenkin and L. H. Ford, Phys. Rev. D26, 1231 (1982); B. Allen, Phys. Rev. D32, 3136 (1985).

[21] A. A. Starobinsky and J. Yokoyama, Phys. Rev. D50, 6357 (1994).

[22] D. Lyth and D. Roberts, hep-ph/9609441.

[23] K. Enqvist, K. W. NG and K. A. Olive, Nucl. Phys. B303, 713 (1988).

[24] A. A. Starobinsky and V. Sahni, in: Proc. 6th Soviet Gravitation Conf., ed. V. N. Ponomarev (MGPI Press, Moscow, 1984); G. Efstathiou and J. R. Bond, Mon. Not. R. Astron. Soc. 218, 103 (1986); H. Kodama and M. Sasaki, Int. J. Mod. Phys. A2, 491 (1987); A. A. Starobinsky, Sov. Astron. Lett. 14, 166 (1988).

[25] A. Linde and V. Mukhanov, Phys. Rev. D 56, 535 (1997), astro-ph/9610219.

[26] P. J. E. Peebles, astro-ph/9805194; astro-ph/9805212.

[27] G. R. Farrar, Phys. Rev. Lett. 76, 4111 (1996); D. J. 
Chung, G. R. Farrar, and E. W. Kolb, astro-ph/9707036.

[28] C. T. Hill, Nucl. Phys. B224, 469 (1983); C. T. Hill, D. N. Schramm and T. P. Walker, Phys. Rev. D36, 1007 (1987); G. Sigl, D. N.Schramm and P. Bhattacharjee, Astropart. Phys. 2, 401 (1994); V. Berezinsky, X. Martin and A. Vilenkin, Phys. Rev. D56, 2024 (1997); V. Berezinsky and A. Vilenkin, astro-ph/9704257.

[29] J. Ellis, J. E. Kim, and D. V. Nanopoulos, Phys. Lett. B145, 181 (1984).

[30] K. Benakli, J. Ellis, and D. V. Nanopoulos, hep$\mathrm{ph} / 9803333$.

[31] T. Han, T. Yanagida and R.-J. Zhang, hep-ph/9804228.

[32] J. W. Cronin, Nucl. Phys. B (Proc. Suppl.) 288, 213 (1992); The Pierre Auger Observatory Design Report (2nd edition), March 1997; M. Boratav, for Pierre Auger Collaboration, The Pierre Auger Observatory Project: An Overview., Proc. of 25th International Cosmic Ray Conference, Durban, v. 5, p. 205 (1997).

[33] S. C. Corbato et al., Nucl. Phys. B (Proc. Suppl.) 288, 36 (1992).

[34] M. Teshima et al., Nucl. Phys. B (Proc. Suppl.) 288, 169 (1992). 